\title{
Civilisations
}

Revue internationale d'anthropologie et de sciences

humaines

48 | 2001

La question de l'islam et de l'Etat à l'aube du XXIe siècle

\section{L'implication des élus français d'origine maghrébine dans la gestion politique des affaires islamiques}

Une réflexion sociologique sur la notion d'« élites laïques à vocation communautaire»

\section{Vincent Geisser}

\section{(2) OpenEdition}

\section{Journals}

Édition électronique

URL : http://journals.openedition.org/civilisations/3472

DOI : $10.4000 /$ civilisations. 3472

ISSN : 2032-0442

\section{Éditeur}

Institut de sociologie de l'Université Libre de Bruxelles

Édition imprimée

Date de publication : 31 août 2001

Pagination : 145-151

ISSN : 0009-8140

\section{Référence électronique}




\title{
L'IMPLICATION DES ÉLUS FRANÇAIS D'ORIGINE MAGHRÉBINE DANS LA GESTION POLITIQUE DES AFFAIRES ISLAMIQUES
}

\author{
UNE RÉFLEXION SOCIOLOGIQUE SUR LA NOTION \\ D' «ÉLITES LAIIQQUES À VOCATION COMMUNAUTAIRE»
}

\section{Vincent GEISSER}

\section{LEDOUBLE PROCESSUS DE POLITISATION ET D'IDÉOLOGISATION DU FAIT ISLAMIQUE EN FRANCE}

Jusqu'au début des années 1980, la gestion politique du fait islamique en France a été dominée par une conception à la fois pragmatique et technocratique. C'estl'État central qui dans le cadre de sa politique générale d'aide aux travailleurs immigrés a encouragé les collectivités locales à mettre en place elles-mêmes des structures spécifiques afin de répondre aux besoins et aux demandes des populations dites «musulmanes» (Gilles Kepel, 1987). Dans ce contexte, certains élus locaux, toutes tendances politiques confondues, ont participé discrètement au financement des premières salles de prière et à la mise en œuvre de «carrés musulmans» dans les cimetières déconfessionnalisés. À l'époque, le fait islamique n'est pas devenu pour autant un enjeu politique local, dans la mesure où il ne suscite encore ni passion, ni fantasme, ni même rejet de la part des élus et de leur électorat.

C'est la crise de l'État central dans sa triple dimension culturelle, sociale et économique qui va contribuer progressivement à hisser le fait islamique au rang de produit politique, faisant l'objet d'une instrumentalisation par les différents acteurs locaux : les maires, l'opposition municipale, les associations de riverains, les associations de commerçants, etc. Le fait islamique acquiert une dimension locale à un moment où il devient également une composante conflictuelle du débat politique national (discussions polémiques sur la légitimité d'une présence musulmane dans l'Hexagone). Ainsi, à partir du milieu des années 1980, il se produit un jeu d'interactions complexes entre le niveau central et les différents échelons locaux et ceci, en totale déconnexion avec les besoins et les demandes cultuelles des populations musulmanes résidant sur le territoire français.

Ce double processus de politisation et d'idéologisation du fait islamique hexagonal aboutit à deux effets majeurs sur le traitement public de l'islam en France.

D'une part, à un abandon des politiques globales, malgré des initiatives de l'État central pour essayer d'organiser sa relation avec les «représentants» du culte musulman : la politique des différents gouvernements, de gauche comme de droite, se limite le plus souvent à quelques 
déclarations d'intention mais n'est suivie d'aucun effet en terme de créations institutionnelles ou de dispositions législatives. L'une des caractéristiques principales du mode de gestion de l'État français en matière d'islam, réside dans cette oscillation permanente entre une tendance interventionniste et une tendance à l'abstentionnisme au nom d'une expression idéologisée de l'idée de laïcité (Franck Frégosi, 1998).

D'autre part, à l'inscription du fait islamique sur l'agenda politique local qui donne naissance à de nouveaux conflits et enjeux : l'islam est désormais perçu par les différents acteurs politiques et associatifs comme un problème local, qu'il convient d'expurger pour certains solution radicale prônée par l'extrême droite -, de réguler pour d'autres, en y apportant des solutions acceptables pour l'ensemble de l'électorat et en développant des médiations avec les communautés musulmanes, représentées abusivement comme des populations à problèmes.

Dans cette nouvelle perspective, où le fait islamique constitue un élément incontournable du débat politique local, les représentants de l'État (les préfets), les maires et les administrations municipales, vont se livrer à la recherche effrénée de médiateurs idéaux, susceptibles de les éclairer dans la mise en œuvre d'une politique musulmane à l'échelon communal.

Dés lors, on comprend l'importance qu'a pu revêtir la présence d'élus français d'origine maghrébine dans les conseils municipaux ${ }^{1}$ : il s'agissait, en quelque sorte d'utiliser au mieux leur origine communautaire-même si cette dernière n'était pas toujours assumée et revendiquée par les acteurs concernés - pour initier des médiations avec les populations musulmanes. On perçoit ici le glissement qui s'est opéré en quelques années. Ces représentants politiques d'origine maghrébine ont été traités par certains notables locaux en «élus musulmans», comme au bon vieux temps de l'Algérie française (syndrome du second collège). Il faut avouer que ce glissement néo-colonial a été largement encouragé par un parti pris laïciste des responsables politiques français qui voyaient d'un mauvais œil l'intervention desélites religieuses musulmanes (imâms ou dirigeants d'associations cultuelles), considérées comme archaïques et trop liées aux États d'origine. Les administrations municipales préfèrent traiter directement avec des élites dites «laïcisées», réputées plus fiables et plus malléables que les élites religieuses. Au départ, l'investiture politique de ces élus français d'origine a donc reposé sur une sorte de paradoxe à la française.

D'un côté, les maires et les institutions locales ont tout fait pour favoriser la promotion d'élites ou de sous-élites communautaires afin de canaliser et d'organiser la demande d'islam, conformément à un vision à la fois policière et sécuritaire.

De l'autre, ils ont évité que ces nouveaux élus d'origine maghrébine apparaissent trop en vue sur le plan communautaire; en ce sens, ces élites ethniques ont été cooptées pour leur capacité à se détacher de leur milieu d'origine et à symboliser les vertus de l'intégration républicaine.

On pourrait résumer en une formule crue cette «consigne politique» donnée par les décideurs locaux à ces nouvelles élites issues de l'immigration maghrébine : sois musulman pour être crédible aux yeux des tiens, mais évite de trop l'être si tu veux conserver ton mandat.

Compte-tenu de ce cahier des charges, les élus d'origine maghrébine ont été contraints de se livrer à un jeu d'équilibriste, jonglant sur le fil du pouvoir local et tenant dans chaque main des ressources particularistes et universalistes. Le moindre faux a provoqué leur disgrâce dans l'espace communautaire comme dans l'espace public.

Dans le cadre de la présente contribution, je développerais l'hypothèse suivante : ces élus français d'origine maghrébine sont d'abord les acteurs publics d'une gestion communautaire qui ne veut pas dire son nom, en raison du tabou républicain (ethnicité républicaine). 
Mais, avant d'exposer les résultats de mon enquête sur l'implication des conseillers municipaux d'origine maghrébine dans la gestion locale du fait islamique, je souhaiterais revenir brièvement sur leur socialisation religieuse et sur leurs représentations de l'islam qui déterminent, en partie, leurs comportements dans l'espace public.

\section{SOCIALISATION RELIGIEUSE ET SYSTĖME DE REPRÉSENTATIONS DU FAIT ISLAMIQUE}

La majorité des élus français d'origine maghrébine se déclarent ouvertement issus de «famille musulmane» : ils n'éprouvent aucune pudeur à avouer la religion de leurs parents, revendiquant leur culture musulmane comme un élément fondamental de leur éducation familiale. Sur ce plan, nous n'avons pas enregistré parmi eux de changement de prénoms ( ex : adoption d'un prénom chrétien) ou même de cas de conversion à la religion catholique. La distanciation à l'égard de la religion du père - «je crois en quelque chose mais plus à la manière de mes parents" - et la critique de certains aspects de l'islam ne signifient pas pour autant un reniement de la religion musulmane. Au contraire, l'islam continue à être vécu comme un patrimoine transmis par les ancêtres qu'il convient à tout prix de préserver.

À travers cette relation à la religion musulmane, nous observons une tendance des élites issues des migrations maghrébines à opérer une idéalisation dans le sens d'un certain conformisme social : «moi, c'est l'islam de France, de la tolérance, l'islam qui s'adapte à toutes les sociétés» (Dahbia, conseillère municipale à Aix-en-Provence, mandat 1989-1995). Elles refusent de considérer leur religion d'origine comme un obstacle à l'intégration et la reconstruisent subjectivement comme un ensemble de valeurs, de justice sociale, de tolérance et d'humanisme. C'est ici que s'affirme de manière saillante une stratégie de réappropriation: «les acteurs s'investissent dans les représentations traditionnelles mais aussi nouvelles (promotion de la femme, libéralisme éducatif, rationalisme scientifique, etc.) en affirmant que si l'on y regarde bien, celles-ci ont toujours été présentes dans le patrimoine originel, soit dans la lettre des textes religieux fondateurs, soit, à défaut, dans ce qu'on tient pour leur esprit» (Carmel Camilleri, 1990 : 103).

Seule, une minorité d'élites d'origine maghrébine développent une vision péjorative du fait islamique, considérant la religion musulmane comme un facteur de régression et d'inadaptabilité à la société française. C'est notamment au sein de cette minorité d'élus que nous avons pu rencontrer les plus farouches opposants au projet de consistoire islamique et, $d$ 'ailleurs, à toute forme d'institutionnalisation du fait islamique dans la société française.

La plupart de ces nouvelles élites sont non-pratiquantes ou pratiquantes occasionnelles. $\AA$ l'exception du jeûne du ramadan, respecté par quelques-unes, les autres pratiques religieuses sont délaissées, voire même ignorées. Généralement, elles n'ont pas reçu d'éducation religieuse, ni au sein de la cellule familiale ni dans les associations ou les amicales contrôlées par les pays d'origine.

Enfin, elles ont tendance à culturaliser leur rapport à l'islam où prédominent les référents festifs et conviviaux sur ceux à caractère proprement religieux : une telle confusion est révélatrice d'une certaine ignorance des préceptes coraniques qui rappelle celle des israélites décrite par Rémy Leveau et Dominique Schnapper : «ils n'en gardent pas moins, par fidélité à soi-même et à un passé familial et collectif, le sentiment d'une identité spécifique, fondée sur le rapport de la tradition [...] dont ils reconnaissent la valeur, malgré leur non-pratique et souvent leur ignorance» (R. Leveau, D. Schnapper, 1984). 
Il convient bien sûr de recourir à cette comparaison avec beaucoup de précautions, sachant que la catégorie israélites connut son apogée à la fin du $\mathrm{XIX}^{\mathrm{c}}$ - début $\mathrm{XX}^{\mathrm{c}}$ siècle, à une époque où le catéchisme républicain et laïque sévissait avec force et tendait à se substituer au catholicisme encore dominant.

Si nous pouvons parler d'élites laïcisées à propos de ces nouveaux élus issus de l'immigration maghrébine, il importe de nuancer cette notion en fonction de l'évolution de sa signification en cours du siècle.

En définitive, les pratiques religieuses et les représentations du fait islamique véhiculées par les élus français d'origine maghrébine n'indiquent pas de disparités fondamentales avec celles observées chez l'ensemble des jeunes issus de l'immigration. Celles-ci peuvent être ramenées à quatre caractéristiques majeures :

- absence de pratique religieuse suivie;

- ignorance des préceptes coraniques;

- confusion totale entre les registres culturels et cultuels;

- volonté commune d'inculquer à leur descendance un libre arbitre («mes enfants, ils choisiront !»).

S'il convient de nuancer la spécificité cultuo-culturelle de ces nouvelles élites politiques locales par rapport au reste des populations d'origine musulmane résidant sur le territoire français, on peut néanmoins s'interroger sur l'incidence de cette «visibilité islamique» sur les conditions de leur investiture politique et le déroulement de leur mandat municipal.

Dans quelle mesure ces élites d'origine maghrébine vont être tentées d'instrumentaliser leur origine musulmane à des fins politico-électorales ?

Quels types de relations entretiennent-elles avec les autres opérateurs islamiques, à savoir les imâms et les dirigeants d'associations cultuelles ?

Dans un contexte où les questions relatives à l'islam font l'objet de débats contradictoires dans l'espace local, les pouvoirs publics ne cherchent-ils pas finalement à faire de ces élus des médiateurs communautaires, au risque de les enfermer dans une logique «d'élus musulmans» au mépris de leur choix et de leur sensibilité personnels?

\section{DES ÉLUS MUSULMANS MALGRÉ EUX?}

À peine élus, certains conseillers municipaux d'origine maghrébine se sont vus confier par leur administration municipale le dossier islam, comme si leur filiation musulmane leur conférait une compétence particulière à traiter ce type de questions. Il faut reconnaître que de nombreux élus ont su jouer habilement sur l'ambiguiité de leur investiture pour se construire à moindres frais une image d'experts en matière de culte islamique et s'imposer comme les intermédiaires obligés entre les pouvoirs publics locaux et les associations musulmanes.

Dans la plupart des municipalités françaises, ces expériences de médiation communautaire se sont soldées par des échecs cuisants, les opérateurs islamiques locaux (imâms, théologiens, présidents d'associations musulmanes) refusant de traiter avec des élus qu'ils considèrent généralement comme des incompétents sur le plan communautaire et religieux.

Il est vrai que ces élus politiques issus de l'immigration maghrébine n'ont généralement qu'une connaissance parcellaire des lieux de culte et des institutions islamiques de leur localité. Cette 
méconnaissance est due, certes, à la complexité des organisations musulmanes, mais apparaît d'abord comme une des conséquences des diverses tentatives d'officialisation engagées par les pouvoirs publics. En effet, les élus ne citent que les lieux de culte, qu'ils qualifient eux-mêmes d'officiels, c'est-à-dire les salles de prière et les associations bénéficiant d'un soutien de la municipalité. Ils opposent ces institutions officielles aux «autres» lieux de culte, auxquels ils accolent abusivement les étiquettes d'obscurantistes et d'intégristes, défendant ainsi une vision manichéenne des réalités islamiques locales, comme s'il y avait d'un côté les bons musulmans, reconnus et soutenus par les pouvoirs publics et de l'autre, les barbus fanatiques, qu'il convient de chasser et d'éradiquer.

À l'exception de quelques échanges de courriers officiels ou d'assistance à des réunions publiques, ces élus d'origine maghrébine entretiennent peu de relations avec les élites cultuelles et religieuses. On peut donc relever une contradiction dans l'attitude de ces élites qui cherchent à contrôler ce qu'elles considèrent comme leur pré-carré communautaire, sans se donner les moyens de s'y investir sérieusement. Il convient également de rechercher les raisons de cette absence d'implication sur le dossier islam dans les représentations croisées que ces différentes catégories d'acteurs ethniques nourrissent l'une à l'égard de l'autre.

Les élites laïques d'origine maghrébine perçoivent les élites cultuelles et religieuses comme les défenseurs d'un certain conservatisme social et comme trop dépendantes des pays d'origine et/ ou des mouvances fondamentalistes.

Quant aux opérateurs islamiques, ils traitent ces élites laïques avec suspicion, les considérant comme des marionnettes politiques sans aucune représentativité communautaire.

Les conseillers municipaux d'origine maghrébine adhèrent rarement à des associations musulmanes sur le plan local comme à l'échelon national. Seule, les organisations musulmanes de la mouvance de la Mosquée de Paris trouvent grâce à leurs yeux (Vincent Geisser, 1997 : 159-164). Ils s'y rendent exceptionnellement pour assister aux assemblées générales ou aux conseils d'administration non pas en tant que membres ordinaires mais souvent en tant que représentants de la mairie.

Enfin, ces élites laïques défendent une conception hygiéniste et sécuritaire de l'islam hexagonal. La majorité d'entre elles véhiculent une représentation manichéenne de l'islam de France, opposant un islam modéré à ce qu'elles appellent un peu rapidement l'intégrisme. Une telle représentation, qui se forge très largement sur les fantasmes et les peurs de la société française, contribue à renforcer le caractère passionnel des débats. Loin d'apaiser les situations paroxystiques, les élus français d'origine maghrébine ont parfois contribué à conforter l'idée d'une menace islamiste sur le territoire français, réduisant ainsi les possibilités d'ouverture et de dialogue avec les élites religieuses et les associations musulmanes. À trop vouloir apparaître comme des élus éclairés, ces élites laïques finissent par accréditer la vision d'une masse musulmane arriérée et tentée par le fondamentalisme islamique. Leur discours emprunte souvent au registre de l'éducation morale.

\section{LES TROIS DILEMMES DE L'ACTION PUBLIQUE DES ÉLITES LAÏQUES EN MATIĖRE D'ISLAM}

L'action publique de ces élites politiques issues de l'immigration maghrébine en matière de culte islamique se heurte à trois dilemmes qui ne sont pas sans rappeler les contradictions auxquelles doivent faire face aujourd'hui les pouvoirs publics français dans leur tentative de définir les contours d'une politique musulmane. 
- Le dilemme local/central ou la difficulté à gérerl'héritage jacobin : les élus français d'origine maghrébine sont unanimes à défendre une conception localiste de la gestion du culte islamique. Mais dans le même temps, ils restent très majoritairement favorables à une option fédérative, calquée sur le modèle du consistoire juif. Leur attachement aux idéaux de la démocratie locale s'accompagne d'un appel permanent à l'État central. À leurs yeux, ce dernier est, d'une part, le garant du droit des minorités face aux abus des pouvoirs locaux et, d' autre part, le protecteur des collectivités musulmanes de France face aux velléités tentaculaires des gouvernements des pays d'origine.

- Le dilemme autonomie/contrôle institutionnel : la référence au dit «modèle juif», moins dans sa version consistoriale, qu'à celle reconstruite sur un mode imaginaire de communauté ou de lobby, traduit leur volonté de se structurer en minorité autonome, exerçant de ce fait une influence sur les pouvoirs publics. Mais ce communautarisme est d' abord un communautarisme tempéré qui prétend s'inscrire dans le cadre des lois et des institutions de la République Française. C'est moins l'appel à l'État en tant qu'organe central qui est évoqué ici que le contrôle du fait islamique par les institutions françaises, qu'elles soient locales, régionales ou nationales.

- Le dilemme visibilitéfinvisibilité du fait islamique ou le complexe de l'islam colonisé : dans leur grande majorité, ces élites politiques d'origine maghrébine ouvrent pour une visibilité locale du fait islamique à travers ses institutions, ses associations et ses lieux de prière. Je citerais cet ancien conseiller municipal d'A vignon : «je crois qu'on vit en Europe, l'islam est à mon avis très beau, parce qu'il peut s'adapter». Cette revendication de visibilité sur le plan local est souvent mêlée à une conception environnementaliste et hygiéniste : les élus font l'apologie d'un islam discret, adapté au cadre urbain, «sans bruit et sans nuisance», que nous qualifierons non sans ironie d'islam propre. Je citerais cet autre élu de Grenoble : «il faut créer des conditions d'environnement immédiat pour qu'il n'y ait pas (...) ce qu'ils [les pouvoirs publics] appellent des rapports conflictuels ou des nuisances, c'est-à-dire par exemple, l'appel à la prière».

\section{VERS UN PŌLE POLITIQUE «ARABO-MUSULMAN» À L'ÉCHELLE EUROPÉENNE?}

La présente contribution atteste d'une dichotomie très nette entre le monde des élites politiques issues des migrations maghrébines, relativement détaché de la sphère religieuse, et le milieu des opérateurs islamiques qui prennent directement en charge les aspects cultuels de l'islam.

Toutefois, la situation a évolué ces dix dernières années aboutissant à une sorte de croisement des itinéraires sociopolitiques : les cadres religieux tiennent de plus en plus un discours citoyen et civique à l'intention de la jeunesse musulmane d'Europe et les cadres politiques sont tentés de s'investir dans les associations musulmanes, conscients des atouts de leur ancrage communautaire.

L'observation des stratégies déployées par les organisations musulmanes en France et en Europe révèle une volonté commune d'inscrire leur combat pour le renouveau de la pensée islamique dans une démarche citoyenne. Il s'agit de vivre son islamité en harmonie avec les sociétés européennes. Loin d'appeler leurs adhérents au repli communautaire, ces organisations les exhortent à réinvestir les valeurs civiques, en faisant d'eux des musulmans respectueux et des électeurs responsables devant les urnes. On voit ici émerger une conception originale qui se manifeste par un désir des dirigeants islamiques d'être des partenaires de premier plan de la socialisation et du passage au politique des nouvelles générations issues de l'immigration maghrébine. 
De leur côté, les élites politiques, longtemps absentes sur le terrain religieux, s'investissent de plus en plus dans des associations et des fédérations musulmanes, revendiquant une sorte de droit de regard laïc sur les questions du culte et l'abattage rituel. Ces élites politiques se considèrent désormais comme des autorités morales de la communauté musulmane, susceptibles de passer des alliances avec les différentes catégories d'opérateurs islamiques (imâms, dirigeants d'associations, présidents de fédérations musulmanes, etc.).

On peut penser que ce croisement des itinéraires (du religieux au politique/du politique au religieux) créera les conditions d'une nouvelle dynamique institutionnelle en Europe qui pourrait déboucher à moyen-terme sur la constitution d'un pôle arabo-musulman à vocation à la fois politique et religieuse.

\section{BIBLIOGRAPHIE}

CAMILLERI, C. 1990. «Identité et gestion de la disparité culturelle : essai de typologie», in Stratégies identitaires, Paris, P.U.F., p. 103.

FRÉGOSI, F. 1998. «Gestion publique de l'islam en France : enjeux politiques, héritage colonial et/ou logique républicaine ?», Correspondances, bulletin scientifique de l'Institut de Recherche sur le Maghreb Contemporain de Tunis, $n^{\circ} 50$.

GEISSER, V. 1997. Ethnicité républicaine : les élites d'origine maghrébine dans le système politique français, Paris, Presses de sciences po, $261 \mathrm{p}$.

KEPEL, G. 1987. Les banlieues de l'Islam, naissance d'une religion en France, Paris, Seuil, 423 p. (collection «À l'épreuve des faits»).

LEVEAU, R. SCHAPPER D. 1984. «Religion et politique : juifs et musulmans maghrébins de France», Revue française de science politique, volume XXVII (6).

\section{NOTES}

1 Lors de notre enquête menée au cours du mandat municipal 1989-1995, nous avons recensé environ 150 conseillers municipaux d'origine maghrébine, élus majoritairement sur des listes d'union de la gauche (Parti socialiste et parti communiste) et plus rarement sur des listes de droite (UDF-RPR). Ces élites locales siégeaient généralement au sein de communes urbaines ou périurbaines dans les grandes régions d'immigration maghrébine, à savoir : Provence-Alpes-Côte d'Azur, Rhône-Alpes, Ile-de-France et Nord. Les femmes représentaient $30 \%$ des nouveaux conseillers municipaux d'origine maghrébine. Enfin, notons que la plupart de ces élus étaient d'origine algérienne (52,8\%), et «harkie» $(20,75 \%)$, les enfants de Tunisiens et de Marocains représentant moins de $15 \%$ de cette population d'élites locales. Pour plus de détails sur la sociologie des élites politiques issues des migrations maghrébine, cf. notre ouvrage Ethnicité républicaine. 